INPLASY

PROTOCOL

To cite: Liu et al. The Efficacy and Safety of Acupuncture Assisted Anesthesia(AAA) for Postoperative Pain of Thoracoscopy: A Protocol for Systematic Review and MetaAnalysis of Randomized Controlled Trials. Inplasy protocol 2021120129. doi: 10.37766/inplasy2021.12.0129

Received: 28 December 2021

Published: 28 December 2021

Corresponding author:

Xinyi Liu

992047152@qq.com

Author Affiliation:

Shandong University of traditional Chinese Medicine

Support: No.

Review Stage at time of this submission: The review has not yet started.

Conflicts of interest:

None declared.

\section{The Efficacy and Safety of Acupuncture Assisted Anesthesia(AAA) for Postoperative Pain of Thoracoscopy: A Protocol for Systematic Review and Meta- Analysis of Randomized Controlled Trials}

$$
\text { Liu, XY1; Meng, D2; Zhao, QY3; Yan, CC4; Wang, JY5. }
$$

Review question / Objective: The aim of this systematic review and meta-analysis of randomized controlled trials is to evaluate the efficacy and safety of Acupuncture Assisted Anesthesia(AAA) on postoperative pain of thoracoscopy. Eligibility criteria: Inclusion criteria: 1.Patients receiving thoracoscopy with acupuncture assisted anesthesia; 2.There is no limitation on the acupuncture measures in the experimental group, include acupuncture, electroacupuncture, transcutaneous electrical acupoint stimulation, et al. 3.The intervention time, frequency, and the electrical stimulation wave of acupuncture is not limited; 4.There is no limitation on the method of anesthesia during surgery, we included both general anesthesia and other anesthesia methods(e.g. PCEA, PCEA or general anesthesia, et al). Exclusion criteria:1.Animal experiments; 2.No acupoints or main and collateral channels are involved.

INPLASY registration number: This protocol was registered with the International Platform of Registered Systematic Review and Meta-Analysis Protocols (INPLASY) on 28 December 2021 and was last updated on 28 December 2021 (registration number INPLASY2021120129).

\section{INTRODUCTION}

Review question / Objective: The aim of this systematic review and meta-analysis of randomized controlled trials is to evaluate the efficacy and safety of Acupuncture Assisted Anesthesia(AAA) on postoperative pain of thoracoscopy.
Condition being studied: Compared with traditional thoracotomy, Video-assisted thoracoscopic surgery (VATS) has certain advantages in reducing surgical trauma, reducing acute and chronic pain and reducing postoperative complications. However, among all surgical operations, even today when VATS is widely used in 
thoracic surgery, the pain after thoracic surgery is still in the forefront of all surgical treatment wounds. The use of opioids will produce more side effects and adverse reactions Good response. So, Enhanced postoperative recovery (ERA) suggests using multimodal analgesia to optimize perioperative pain management. More and more studies show that acupuncture assisted anesthesia (AAA) has become an important part of multimodal non opioid analgesia, which will help to promote postoperative enhanced recovery (ERA) improvement of the scheme. In recent years, acupuncture related technologies such as percutaneous acupoint electrical stimulation, electroacupuncture and lateral ankle acupuncture combined with anesthesia have been applied to vats, which has significant advantages in postoperative analgesia, protecting organ function and reducing inflammatory reaction. However, there is a lack of systematic evaluation research on the safety and effectiveness of acupuncture assisted anesthesia on pain after thoracoscopic surgery. Therefore, relevant research has been carried out This study is of great significance to further promote the application of acupuncture assisted anesthesia in thoracoscopic surgery.

\section{METHODS}

Search strategy: We will search articles in three electronic database including PubMed, EMBASE and Cochrane Library and four Chinese databases (China National Knowledge Infrastructure, Chongqing VIP Information, and WanFang Data, Chinese Biomedical Database). All the English publications until 30 November 2021 will be searched without any restriction of countries or article type. Reference list of all selected articles will independently screened to identify additional studies left out in the initial search. We also further searched the grey literature and the retrieved references to avoid omission. For the literature to be difficult to obtain the full text, we checked and identify the ongoing or unpublished studies through the World Health Organization International Clinical Trials
Registry Platform (WHO ICTRP), ClinicalTrials.gov, Chinese Clinical Trial Registry (Chi CTR), and the reference list of eligible RCTs. We performed an initial search of PubMed as follows(Table 1): \#1 ( (()(()((lacupuncture[MeSH Terms]) OR (acupuncture therapy[Title/Abstract])) OR (electroacupuncture[Title/Abstract])) OR (ear acupuncture[Title/Abstract])) OR (auricular acupuncture[Title/Abstract])) OR (TEAS[Title/Abstract])) OR (transcutaneous electrical acupoint stimulation[Title/ Abstract])) OR (acupuncture point[Title/ Abstract])) OR (acupoint[Title/Abstract])) OR (acupressure[Title/Abstract])) OR (moxibustion[Title/Abstract]); \#2 (()(()((lanaesthesia[MeSH Terms]) OR (narcosis [Title/Abstract])) OR (narcotism[Title/Abstract])) OR (anaesthetization [Title/Abstract])) OR (analgesia[Title/Abstract])) OR (general anesthesia[Title/Abstract])) OR (nerve block[Title/Abstract])) OR (patient controlled epidural analgesia[Title/ Abstract])) OR (patient controlled intravenous analgesia[Title/Abstract])) OR (PCEA[Title/Abstract])) OR (PCIA[Title/ Abstract]); \#3 (((thoracoscopy[MeSH Terms]) OR (thoracoscopic surgery[Title/ Abstract])) OR (VATS[Title/Abstract])) OR (video-assisted thoracoscopic surgery[Title/Abstract]); \#4 ((()((Pain, Postoperative[MeSH Terms]) OR (Pain, Postoperative[Title/Abstract])) OR (pain management[Title/Abstract])) OR (postoperative analgesi`[Title/Abstract])) OR (pain management[Title/Abstract])) OR (ache*[Title/Abstract])) OR (suffering*[Title/ Abstract])) OR (discomfort[Title/Abstract]).

Participant or population: Inclusion criteria: 1.Patients receiving thoracoscopy; 2.There is no limitation on sex, age, race, disease category, et al. 3.There is no limitation on the method of anesthesia during surgery, we included both general anesthesia and other anesthesia methods. Exclusion criteria: 1. The adjacent nerves of acupoints, such as median, ulnar and radial nerves, are confirmed to have impaired function and hypoesthesia due to cervical spondylosis or trauma; 2.There is local infection or chronic inflammation on the surface of the acupoint stimulation site, or 
the electrode cannot be applied; 3.Severe pulmonary infection, bronchitis, airway hyperresponsiveness and large amount of expectoration are expected to have a great impact on postoperative extubation; 4.Patients with severe central system diseases, rheumatic immune system diseases and severe mental diseases before operation; 5.Patients who have participated in other clinical trials in recent 4 weeks; 6. Bronchodilation, pulmonary tuberculosis and severe pleural adhesion are not suitable for thoracoscopic surgery;7.Patients who cannot cooperate to complete the study plan, including those with language difficulties, infectious diseases or other medical history.

Intervention: Inclusion criteria: 1.Patients receiving thoracoscopy with acupuncture assisted anesthesia; 2. There is no limitation on the acupuncture measures in the experimental group, include acupuncture, electroacupuncture, transcutaneous electrical acupoint stimulation, et al.3.The intervention time, frequency, and the electrical stimulation wave of acupuncture is not limited; 4.There is no limitation on the method of anesthesia during surgery, we included both general anesthesia and other anesthesia methods(e.g. PCEA, PCEA or general anesthesia, et al). Exclusion criteria: 1.Animal experiments; 2.No acupoints or main and collateral channels are involved.

Comparator: Inclusion criteria:The control group underwent mock-acupuncture or blank control or do not combined acupuncture with anesthesia(such only uses anesthesia, or only uses acupuncture) Exclusion criteria: Compared with the treatment group, underwent different frequency, waveform, intervention time, or other stimulation factor of acupuncture.

Study designs to be included: Randomized controlled trials.

Eligibility criteria: Inclusion criteria: 1.Patients receiving thoracoscopy with acupuncture assisted anesthesia; 2.There is no limitation on the acupuncture measures in the experimental group, include acupuncture, electroacupuncture, transcutaneous electrical acupoint stimulation, et al. 3.The intervention time, frequency, and the electrical stimulation wave of acupuncture is not limited;

4.There is no limitation on the method of anesthesia during surgery, we included both general anesthesia and other anesthesia methods(e.g. PCEA, PCEA or general anesthesia, et al).Exclusion criteria:1. Animal experiments; 2.No acupoints or main and collateral channels are involved.

Information sources: We will search articles in three electronic database including PubMed, EMBASE and Cochrane Library and four Chinese databases (China National Knowledge Infrastructure, Chongqing VIP Information, and WanFang Data, Chinese Biomedical Database). All the English publications until 30 November 2021 will be searched without any restriction of countries or article type. Reference list of all selected articles will independently screened to identify additional studies left out in the initial search. We also further searched the grey literature and the retrieved references to avoid omission. For the literature to be difficult to obtain the full text, we checked and identify the ongoing or unpublished studies through the World Health Organization International Clinical Trials Registry Platform (WHO ICTRP), ClinicalTrials.gov, Chinese Clinical Trial Registry (Chi CTR), and the reference list of eligible RCTs.

Main outcome(s): The aim of this systematic review and meta-analysis of randomized controlled trials is to evaluate the efficacy and safety of Acupuncture Assisted Anesthesia(AAA) on postoperative pain of thoracoscopy. 1.There are no restrictions on the duration of acupuncture and the timing of its implementation (preoperative, intraoperative, and postoperative). Therefore, subgroup analysis should consider whether acupuncture can be divided according to the duration of acupuncture (long-term, 
short-term) and the implementation timing (before, during and after the operation). Considering that this factor may be the source of heterogeneity, different action time may have different effects on postoperative pain. 2.According to the VAS scores at different time points after operation, the effect of acupuncture on postoperative pain was evaluated. 3.The effect of acupuncture on postoperative pain was evaluated according to the amount of postoperative analgesia pump or analgesia analgesic. 4.Predicting the effect of waveform and frequency of acupuncture on postoperative pain. Predict and find out the relatively optimized acupuncture method, so as to assist anesthesia to enhance the control of postoperative pain.

Quality assessment / Risk of bias analysis: The "process-based evaluation table" attached to the Cochrane Collaboration Network was used to test the quality of literature. Selection risk: to judge the accuracy of the randomized scheme and the concealment of the allocation scheme; implementation risk: to judge whether the blinding of the participants is perfect; measurement risk: to judge whether the analyst blinding is successful; follow up bias: whether the results are reported completely, such as loss of follow-up, exit, and other information; reporting risk: the possibility of selective reporting; other bias: judging whether there are other factors High risk of bias. When the number of studies was more than 10, a funnel plot was drawn to analyze publication bias. If there was significant publication bias, the influence of publication bias on outcome indicators was evaluated by Egger's test; if the number was less than 10 , publication bias analysis was not conducted.

Strategy of data synthesis: Meta-analysis was carried out based on Stata 15.0 software. Relative Risk(RR)/Odds Ratios(OR) was used for secondary classification data, and weighted mean difference (WMD) or standard mean difference (SMD) was used to combine effect values for continuous variables, and corresponding $95 \%$ confidence interval $(95 \% \mathrm{Cl})$ was calculated.

Subgroup analysis: 1.There are no restrictions on the duration of acupuncture and the timing of its implementation (preoperative, intraoperative, and postoperative). Therefore, subgroup analysis should consider whether acupuncture can be divided according to the duration of acupuncture (long-term, short-term) and the implementation timing (before, during and after the operation). Considering that this factor may be the source of heterogeneity, different action time may have different effects on postoperative pain. 2.According to the VAS scores at different time points after operation, the effect of acupuncture on postoperative pain was evaluated. 3.The effect of acupuncture on postoperative pain was evaluated according to the amount of postoperative analgesia pump or analgesia analgesic. 4.Predicting the effect of waveform and frequency of acupuncture on postoperative pain. 5.Predict and find out the relatively optimized acupuncture method, so as to assist anesthesia to enhance the control of postoperative pain.

Sensitivity analysis: Meta-analysis was carried out based on Stata 15.0 software. Relative risk (RR) or odds ratio (OR)was used for secondary classification data, and weighted mean difference (WMD) or standard mean difference (SMD) were used to combine effect values for continuous variables, and corresponding $95 \%$ confidence interval (95\% CI) was calculated. When $p>0.10$ and $12<50 \%$, fixed effect model was selected; when $p<$ 0.10 or $12>50 \%$, random effect model was selected, and 12 had higher priority than $\mathrm{p}$ value. Test level $a=0.05$. When heterogeneity exists, the first review whether the data extraction is correct, draw a Galbraith diagram to verify the heterogeneity, explore the source of heterogeneity, continue to use subgroup analysis or meta-regression, and determine the stability of the effect value by sensitivity analysis after eliminating the heterogeneity points. If the heterogeneity is very 
significant, meta-analysis is not appropriate, and only a qualitative description is made. When the number of studies was more than 10 , a funnel plot was drawn to analyze publication bias. If there was significant publication bias, the influence of publication bias on outcome indicators was evaluated by Egger's test; if the number was less than 10 , publication bias analysis was not conducted.

Country(ies) involved: China.

Keywords: Efficacy;Safety;Acupuncture Assisted Anesthesia(AAA); Postoperative Pain in Thoracoscopy; Protocol; Systematic Review and Meta-Analysis of Randomized Controlled Trials.

Contributions of each author:

Author 1 - Xinyi Liu found the research direction and drafted the manuscript.

Email: 992047152@qq.com

Author 2 - Dan Meng provided themethods of statistical analysis.

Email: mengdan1993@sina.com

Author 3 - Qinyu Zhao contributes to the formulation of selection criteria and the risk of deviation evaluation strategy.

Email: Qinyu Zhao

Author 4 - Chunchun Yan read, provided feedback, and approved the final manuscript.

Email: yanchunchun1986@163.com

Author 5 - Jingyu Wang involved a preliminary subgroup analysis scheme.

Email: 24813694@qq.com 